\title{
Proposal and analysis of integrated PTN architecture in the mobile backhaul to improve the QoS of HetNets
}

\author{
David Cortés-Polo ${ }^{*}$, José-Luis González-Sánchez ${ }^{1}$, Javier Carmona-Murillo² and Franciso J Rodríguez-Pérez²
}

\begin{abstract}
The rapid progress made in mobile device technologies has implied that the access network must evolute and develop new strategies to satisfy the requirements of the users. Heterogeneous network (HetNet) allows for a flexible deployment strategy and offers economically viable solutions to improve network scalability and indoor coverage. This emerging topic has caught the attention of the research community and the industry because of the importance of these networks to satisfy the demand of data services. To provide this demand, different parameters of quality of service (QoS) must be satisfied. In this paper, we present a study on recent advances and open research issues on Mobility Protocols in conjunction with Multi-Protocol Label Switching (MPLS)-based packet transport networks (PTN) to provide QoS in wireless heterogeneous networks. Various mobile management protocols and their interaction with the mobile backhaul and packet core network are briefly introduced. A new architecture called Integrated Proxy Mobile MPLS-TP (IPM-TP) is also outlined to reduce the signalling cost and improve the QoS in HetNets with high rates of mobility.
\end{abstract}

Keywords: Mobile backhaul; Proxy mobile IPv6; MPLS-TP; PTN; Signalling cost; Network convergence; Seamless mobility management

\section{Introduction}

With the appearance of smart phones and the emergence of heterogeneous interconnected systems, the demand for new mobile broadband is growing exponentially. New data services and applications developed, requiring significant increases in the network capacity not only in the radio access network (RAN) but also in the aggregation and the core network.

According to a recent forecast, global mobile data traffic has grown $81 \%$ in 2013 , and this traffic will increase 11 fold between 2013 and 2018 [1]. This increment requires new deployment in the radio access network in order to satisfy these network connectivity requirements by means of increasing spectral efficiency, building new cells, splitting cells, and deploying small cells. These mechanisms

*Correspondence: david.cortes@cenits.es

1 Research, Technological Innovation and Supercomputing Center of Extremadura (CénitS), National Road 521, km 41.8, Postal code 10.071 Cáceres, Spain

Full list of author information is available at the end of the article construct a multi-tier network called the heterogeneous network (HetNet) [2].

Whereas a traditional homogeneous cellular system is a network of base stations transmitting at similar power levels, HetNets allow for a flexible deployment strategy with the use of different power base stations including femtos, metros, picos, relays, and macros to provide coverage and capacity where it is needed the most.

To support this integration, an IP-based backhaul is deployed in the mobile network, and it is integrated with the packet core network. This is a flat all-IP-based network which supports not only the services deployed in the mobile network but also the handover between heterogeneous technologies seamlessly [3].

With this architecture, the integration of heterogeneous networks aimed by the fourth-generation (4G) wireless networks is obtained in order to satisfy the increasing demand of users in terms of quality of service (QoS) and bandwidth requirements [4,5].

To provide QoS and satisfy bandwidth requirements of the mobile communications, the mobile packet core

\section{Springer}

(c) 2015 Cortés-Polo et al.; licensee Springer. This is an Open Access article distributed under the terms of the Creative Commons Attribution License (http://creativecommons.org/licenses/by/4.0), which permits unrestricted use, distribution, and reproduction in any medium, provided the original work is properly credited. 
network and the backhaul must implement a control plane which handles and tracks the mobility of the mobile node $(\mathrm{MN})$ and also maintains the active services when the handover is produced.

The 3rd Generation Partnership Project (3GPP) has defined a proposal which implements Proxy Mobile IPv6 (PMIPv6) [6] in the Evolved Packet Core (EPC) to track the mobility and keep the connectivity with the mobile node [7]. This proposal tracks the movements of the MN and initializes the mobility signalling in order to set up the required routing state. This reduces the signalling of the mobile node and the complexity of the protocol stack.

The industry have also developed architectures to facilitate interworking between fixed network and the wireless networks [8]. These architectures allow the MN to move between the mobile network and the fixed broadband network and vice versa as well as dual access WAN residential gateway. However, this approach presents some drawbacks, such as long handoff latency or large signalling load due to frequent registration updates when the MN velocity is high.

Taking into account this scenario, there are three important issues that must be resolved to accommodate the mobile data traffic into these networks: the integration of heterogeneous networks, the network connectivity maintenance and the resource provisioning required by the MN.

The first issue is addressed by $4 \mathrm{G}$ wireless networks which aim to integrate heterogeneous networks seamlessly in order to satisfy the increasing demand of the mobile users.

The IP-based mobility management protocols solve the second issue which is the network connectivity maintenance, but the resource provisioning is not resolved to maintain the requirements of the MN.

To take advantage of this development, improve the convergence between the fixed and mobile network and resolve the increasing resource-provisioning requirements of the $\mathrm{MN}$, a new architecture called Integrated Proxy Mobile MPLS-TP (IPM-TP) is proposed. This architecture increases the path-protection mechanisms and supports dynamic topology changes and network optimization produced by the movement of the mobile nodes in HetNets.

The remainder of this paper is organized as follows. Section 2 introduces the related works. Section 3 introduces the proposed architecture designed for enhancing QoS of the mobile device by reducing the signalling cost and preventing data packet loss. In Section 4, analytical models are presented. Section 5 presents the performance evaluation to derive the handover signalling overhead, total cost rate and the service-blocking probability required for all underlying protocols. The results are given in Section 6. Finally, Section 7 contains the concluding remarks.

\section{Related works}

HetNets provide two basic benefits to operators: they increase capacity in hotspots and improve coverage in places where macro coverage is not adequate.

One of the challenge of these HetNets is to provide the QoS required by the MNs. This is a key aspect because of the small cell technologies enumerated in the previous section, and the backhaul technologies that interconnect the small cells with the core network must provide this QoS. There are various approximations to deploy the small cell backhaul.

- Macrocell as an aggregation point. The small cell uses the macro backhaul facility to get to the core network.

- Fixed backhaul. The small cell backhaul is provided by a wireline network.

Figure 1 shows different interconnections between the small cells and the backhaul. As can be observed, macrocell as aggregation point (1) and fixed backhaul (2) approaches are described in the figure. Those backhauls interconnect the core packet network with the MN and must provide and maintain IP connectivity to the mobile nodes.

Proxy Mobile IPv6 is the protocol chosen to track the movements of the $\mathrm{MN}$ and initialize the mobility signalling in order to set up the required routing state $[7,9]$.

This protocol introduces two functional entities to the access network, the Mobile Access Gateway (MAG) and the Local Mobility Anchor (LMA). The MAG typically runs on the access router (AR). The main role of the MAG is to detect the movements of the MN and initiate mobility-related signalling with the LMA on behalf of the MN.

In addition, the MAG establishes a tunnel with the LMA for enabling the MN to use an address from its home network prefix and emulates the home network of the $\mathrm{MN}$ on the access network for each MN.

On the other hand, the LMA ensures that the MN address remains reachable while it moves, stores the information necessary to associate an $\mathrm{MN}$ with its serving MAG and enables the relationship between the MAG and LMA to be maintained.

Figure 2 shows the basic PMIPv6 domain with heterogeneous technologies.

Therefore, backhaul and packet core network performance are critical for end-to-end mobile-network performance. Key metrics such as throughput, latency and jitter are important, and the operators maintain these metrics in the mobile communication [10]. 


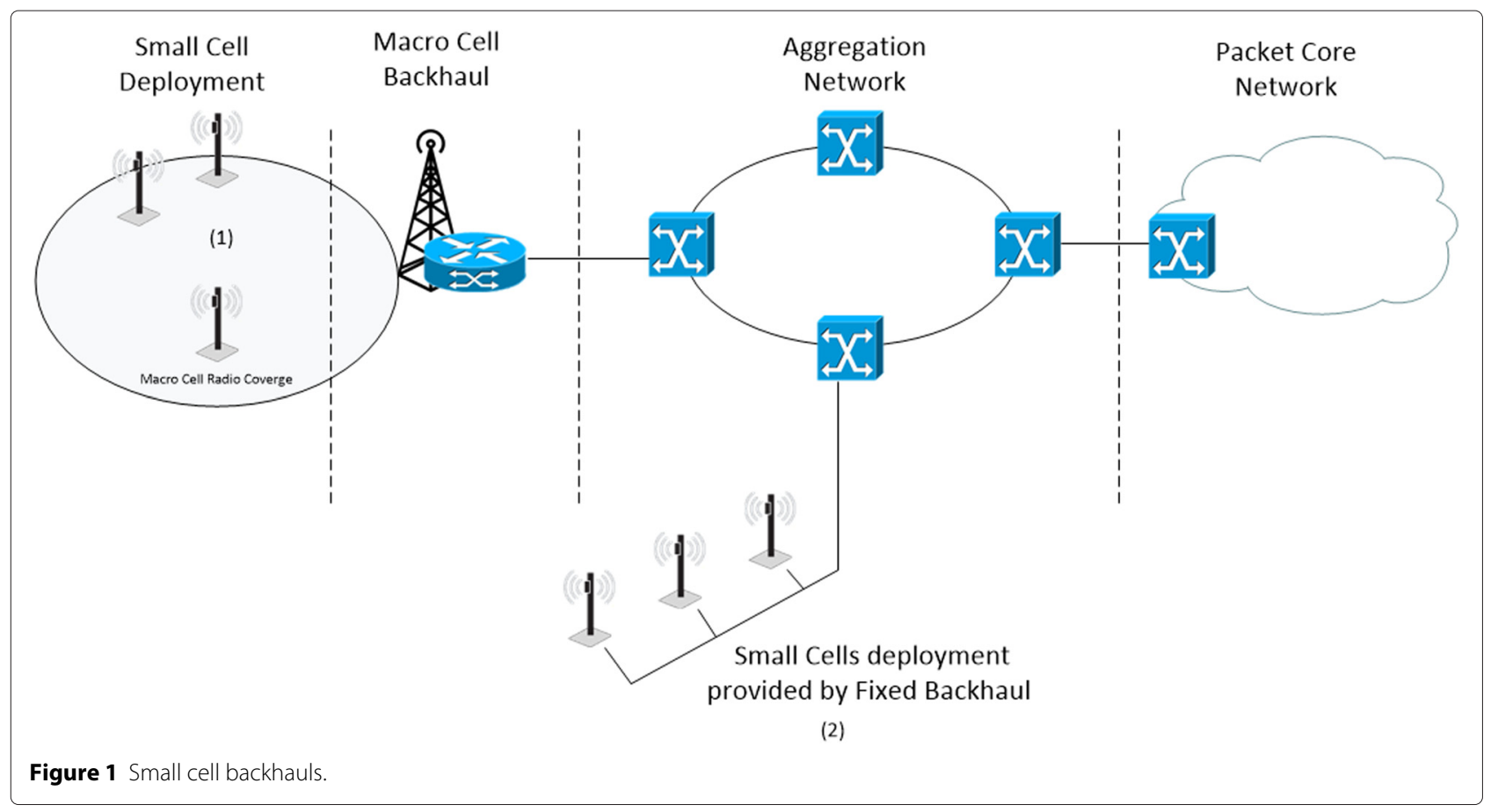

In this sense, traditional E1/T1 and ATM-based backhauls and access networks deployed in 2G and 3G are not viable because of the cost associated to adapt those technologies to the new requirements such as QoS, timing synchronization, lower packet loss and high availability [11].
Network operators looks for flexibility, scale and operational simplicity to lower the total cost of ownership and simultaneously enhance the mobile service. In this sense, the IP/MPLS network deployed in the mobile backhaul and the core packet network provides higher resiliency and ensures the reliability of the mobile backhaul [12].

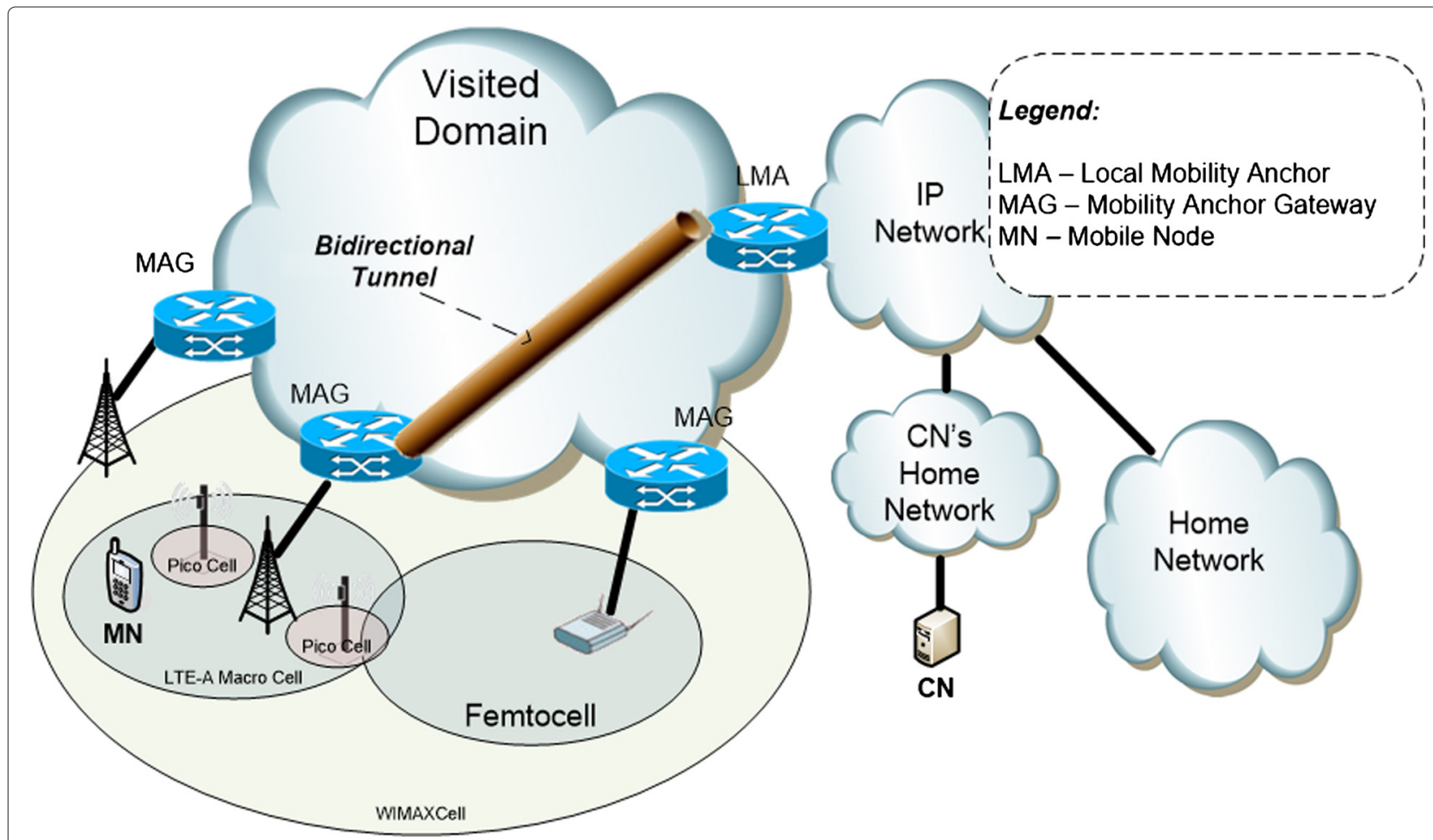

Figure 2 PMIPv6 domain with HetNets integrated in the domain. 
Some approaches integrate the IP-based mobily protocol with the Multi-Protocol Label Switching (MPLS) technology implemented in the mobile backhaul and the core packet network. One of these approaches is MPLS tunnel support for the Proxy Mobile IPv6 (PMIP-MPLS) [13]. PMIP-MPLS implements the same architecture as a PMIPv6 domain maintaining the MAGs and LMAs entities with the same functionalities.

The main difference between both protocols is the tunnelling mechanism used to deliver the packets from the LMA to the MAG. The IETF advises the use of IP-inIP or Generic Routing Encapsulation (GRE) as tunnelling methods.

IP-in-IP is a protocol by which an IP datagram may be encapsulated (carried as payload) within an IP datagram, by adding a second IP header to each encapsulated datagram. GRE is another tunnelling method that encapsulates any network layer packet. GRE requires an IP-in-IP header to encapsulate the information and also a GRE header to be added to the packet.

With the MPLS tunnel, the overhead added by the tunnelling method decreases due to the MPLS label size, which is 15 times less than a traditional IP-in-IP or GRE header needed by the tunnel (i.e. MPLS label size is 4 bytes in length whereas the IP header size is 20 bytes in length).

However, this approach presents similar drawbacks like PMIPv6, such as long handoff latency or large signalling load due to frequent registration updates.

Nowadays, many fixed network carriers have moved from IP/MPLS approaches towards Multi-Protocol Label Switching - Transport Profile (MPLS-TP) as the protocol to converge the traditional fixed networks with the packet-based transport networks [14]. MPLS-TP is based on the same forwarding mechanisms as MPLS but has enhanced Operations, Administration and Maintenance (OAM) and protection capabilities, allowing it to become a true Carrier Class Transport Network Technology and achieve a higher efficiency and a lower operational cost, while maintaining transport characteristics [15].

The next section describes the functionality of the proposed IPM-TP approach which integrates the fixed network based in packet transport network and the mobile network reducing the signalling overhead, including path protection mechanisms, supporting dynamic topology changes and introducing resiliency capabilities in the presence of high-mobility scenarios.

\section{Integrated Proxy Mobile MPLS-TP}

In this section, the new approach called IPM-TP is presented. It integrates the wireless heterogeneous networks and the fixed packet transport network supporting dynamic topology changes and QoS provision to the network.
We assume that an MPLS-TP packet core network exists between the Ingress Label Edge Router/Local Mobility Anchor (Ingress LLMA) and the Egress Label Edge Router/Mobility Anchor Gateway (Egress LMAG). The Ingress LLMA performs the role of an edge LER, filtering between intra- and inter-domain signalling. At the same time, this network element has the functionality of a LMA. The LMAG is connected to several access points (APs) that offer link-layer connectivity.

We also distinguish here between link-layer functionalities of the air interface, which are handled by the AP, and IP-layer mobility (L3 handoff), which occurs when the MN moves between subnets served by different LMAGs. Note that an LMAG is the first IP-capable network element seen from the mobile node. Thus, the LMAG also performs the role of an Egress LER. Figure 3 shows the behaviour of IPM-TP.

Messages 1 and 2 are produced when the mobile node detects the handover and notifies it to the serving gateway (LMAG1). The LMAG initializes the handover in the network sending a handover initiate (HI) message.

Messages 3 to 5, the LMAG1 tracks the mobility of the mobile node.

The LMAG1 chooses the best path and extends the label switched path (LSP) employing the usual MPLS-TP using the RSVP-TE protocol. The new LSP segment forwards all the packets sent to the mobile node which will be attached in LMAG2. This LSP is created because the MN reports the ID of the LMAG to attach to.

Data packets are sent from the LLMA through the LLMA-LMAG1 tunnel and forwarded using the LMAG1LMAG2 tunnel. The packets will be buffered in LMAG2 until the mobile node is attached. This buffering technique prevents the packet loss. A Handover ACK (HAck) message is sent to the LMAG1 to notify that the network is prepared to support the handover.

Messages 6 and 7 introduce the L2 signalling in the network. These messages notify the mobile node that the new wireless network, which it is going to attach to, is prepared.

Messages 8 to 10 handle the location update of the mobile node in the LLMA. When the QoS of a particular flow is under an agreed threshold assigned in the service level agreement (SLA), or the negotiation using RSVP resource reservation is not satisfied, the LMAG updates the location with the LLMA. This is measured by means of the OAM capabilities included in MPLS-TP [14].

The LMAG sends a PBU message to update the binding cache of the LLMA. When this message is received, a new tunnel is created using RSVP PATH/RESV messages. Finally, a PBAck message is sent to the LMAG2 to confirm the location update as is described in PMIPv6 RFC. 


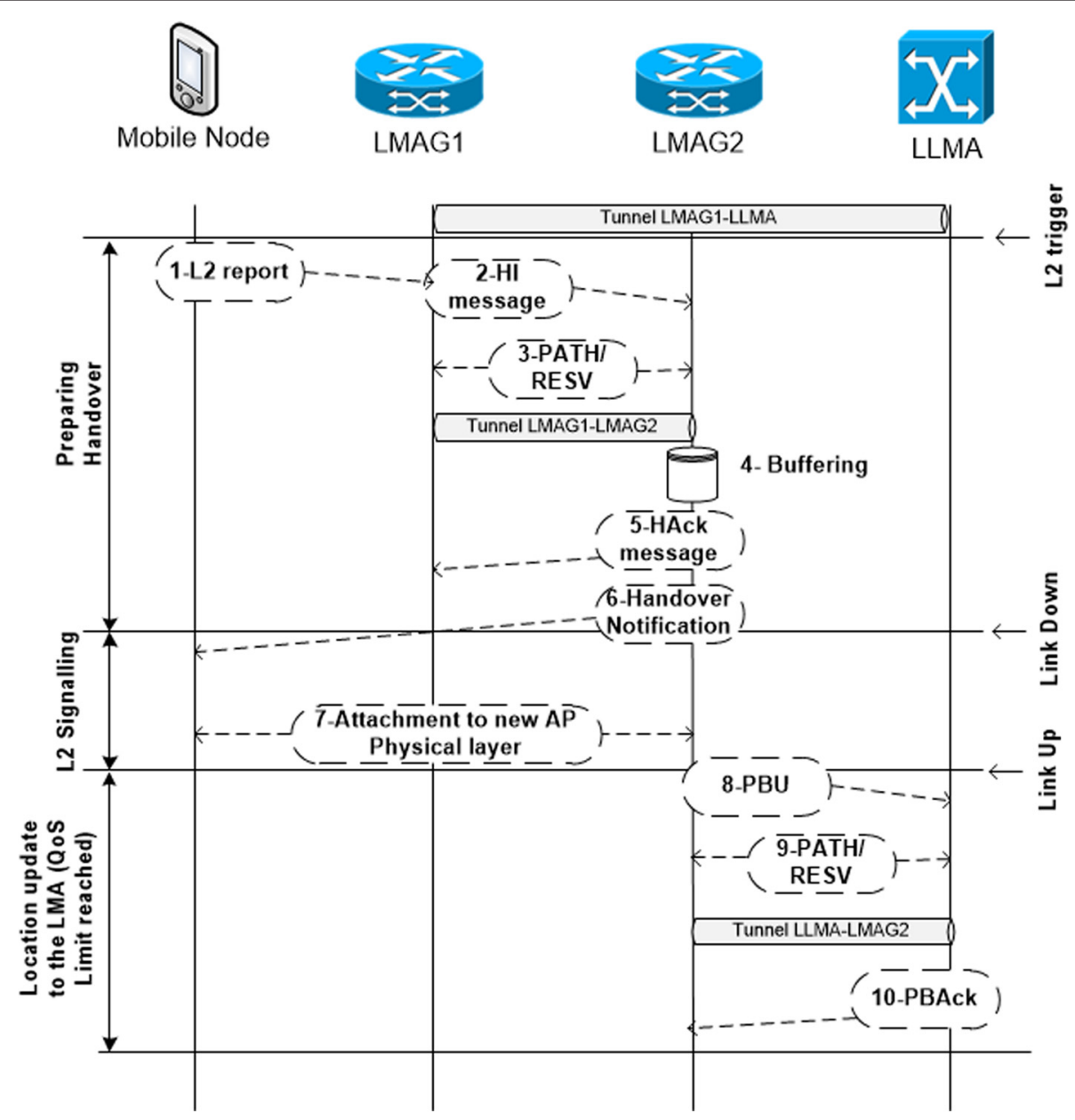

Figure 3 Handover process proposed in IPM-TP. The original tunnel from LMA to MAG1 is extended to MAG2 using a new segment of label switched path (LSP).

\section{Analytical models}

In this section, the analytical model including network, mobility and traffic model is described. This analytical model will be used in the performance evaluation.

\subsection{Network model}

The domain is composed of $N$ cells connected to different MAGs which are the first IP-capable device in the network.

For the simplicity of the model, we assume that mobile service areas are partitioned into areas of the same size for both macrocell and microcell as described in [16]. These areas have circular shape with the same area $S$. The cell size will be calculated as $S=\pi R^{2}$, where $R$ is the radius.

Our scheme works both in the macrocell and the microcell environment. We note that, if the size of cells is small, the probability of movement will be high and vice versa [17].
The domain topology is depicted in Figure 4. Let $d_{x, y}$ the hop distance between two nodes $(x, y)$ in the network.

\subsection{Mobility and traffic models}

The mobility model represents the movement of the MN. The model is based on the well-known fluid flow (FF) model [18]. Under this model, it is assumed that the direction of the movement is uniformly distributed over the range $(0,2 \pi)$ and that the $\mathrm{MN}$ is with high mobility and infrequent speed change.

The following assumptions are made to derive the mobility and traffic models.

- The session arrival to the MN follows a Poisson distribution with rate $\lambda_{s}$.

- The residence time in a cell follows an exponential distribution. 


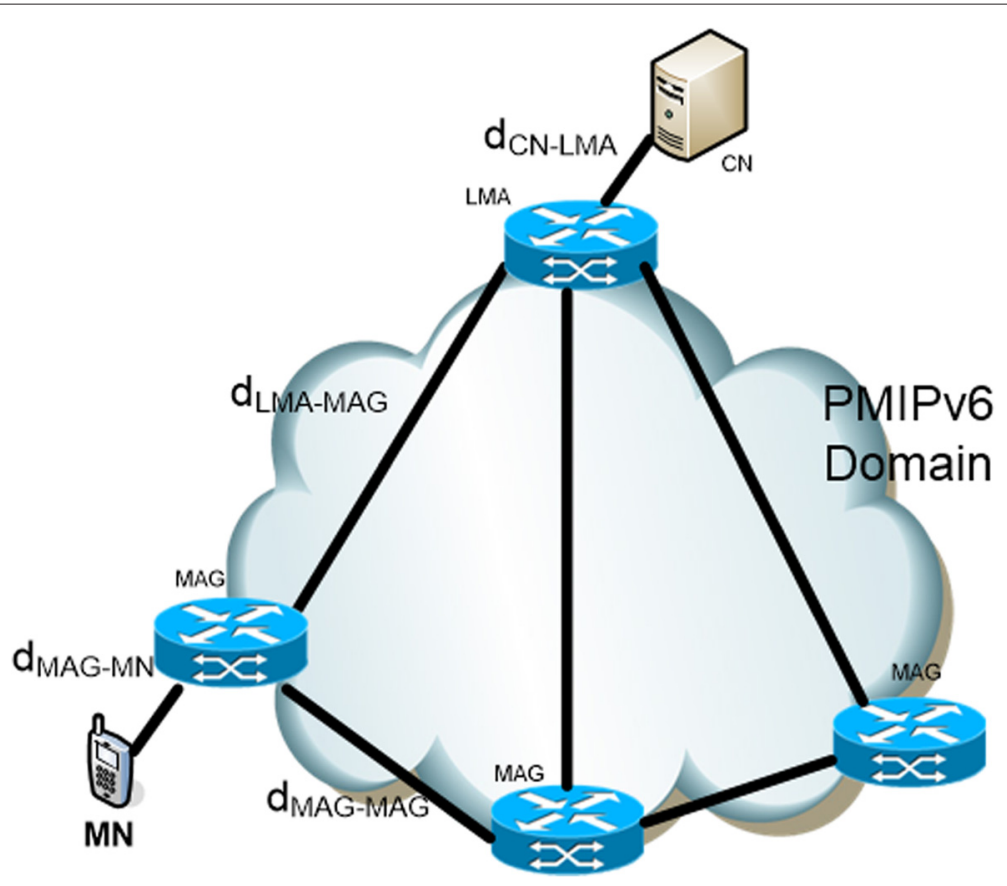

Figure 4 Domain topology.

The motivation behind these choices is the memoryless property of the exponential distribution which can represent the human behaviour.

Besides, these are the chosen distributions in the majority of literature studies using fluid flow to characterize the mobility in a model [16,19-22].

Let $\mu_{c}$ the cell-crossing rate [23]. It can be expressed as:

$$
\mu_{c}=\frac{2 v}{\sqrt{\pi S}}
$$

where $v$ is the average velocity of the $\mathrm{MN}$.

From the assumptions made above and Equation 1, the average number of movements during an intersession arrival $N_{h v}$ can be calculated as follows:

$$
N_{h v}=\frac{\mu_{c}}{\lambda_{s}}
$$

\section{Performance evaluation}

PMIPv6, MPLS support for PMIPv6 and IPM-TP approaches are compared through analytical models to derive the signalling cost, the packet delivery cost and the service-blocking probability.

These analytical models are derived using the framework described in [24]. The packet transmission cost in IP networks is proportional to the distance in hops between source and destination nodes.
Thus, the transmission cost of a packet (signalling or data) between nodes $X$ and $Y$ belonging to the wired part of a network can be expressed as $C$ (wired) $=\alpha$. $\operatorname{Size}($ Packet $) \cdot d_{X-Y}$ while $C$ (wireless) $=\beta \cdot \operatorname{Size}($ Packet $)$. $d_{M N-Y}$, where $\alpha$ is the unit transmission cost over wired link and $\beta$ the weighting factor for the wireless link.

\subsection{Signalling cost}

Signalling cost of registration updates during a session is denoted by $C_{u}(\cdot)$, where $(\cdot)$ is one of the studied protocols (PMIPv6, PMIP-MPLS or IPM-TP). The signalling cost is the accumulative traffic load on exchanging signalling messages during the communication session of the $\mathrm{MN}$.

For each movement into a new subnet, the Proxy Binding Update message is sent to the LMA. This mechanism is performed in both PMIPv6 and PMIP-MPLS. In IPMTP, a LSP tunnel extension is created between the previous LMAG and the new LMAG to forward the packets to the mobile node. This LSP tunnel is extended while the QoS requirements are satisfied. The registration with the LLMA will be done when the threshold requirements are reached (i.e. the bandwidth or the delay is not satisfied).

Let $C_{u}$ (PMIPv6) be the signalling cost of the Proxy Mobile IPv6 protocol. Then, it can be expressed as:

$$
C_{u}(\mathrm{PMIPv} 6)=N_{h v}[C(\mathrm{PBU})+C(\mathrm{PBA})]
$$

Equation 3 illustrates the behaviour of the PMIPv6 handover mechanism described in [6]. When the MN 
moves from a MAG to another, the serving MAG sends a Proxy Binding Update message (PBU) to notify the handover to the LMA. This notification has a registration cost, denoted by $C(\mathrm{PBU})$ in the equation, that is composed of the transmission cost of the packet, the routing table lookup and the registration update processing in the entities involved in the mobility process.

To acknowledge the update, the LMA sends a Proxy Binding ACK (PBA) to the serving MAG where registration cost is denoted by $C$ (PBA).

To obtain the signalling cost of a session, all PBU/PBA messages must be considered. This is denoted by the $N_{h v}$ term in the equation.

The PBU and PBA registration cost is denoted by $C(\mathrm{PBU})$ and $C(\mathrm{PBA})$, respectively, and can be derived as follows:

$$
\begin{aligned}
C(\mathrm{PBU})= & \alpha\left(S(\mathrm{PBU}) d_{\mathrm{MAG}-\mathrm{LMA}}\right)+\left(d_{\mathrm{MAG}-\mathrm{LMA}}-1\right) \mathrm{PC}_{r} \\
& +\mathrm{PC}_{\mathrm{LMA}}
\end{aligned}
$$

$$
\begin{aligned}
C(\mathrm{PBA})= & \alpha\left(S(\mathrm{PBA}) d_{\mathrm{LMA}-\mathrm{MAG}}\right)+\left(d_{\mathrm{LMA}-\mathrm{MAG}}-1\right) \mathrm{PC}_{r} \\
& +\mathrm{PC}_{\mathrm{MAG}}
\end{aligned}
$$

where $\alpha$ is the transmission unit cost in a wired network, and $\mathrm{PC}_{r}, \mathrm{PC}_{\mathrm{LMA}}$ and $\mathrm{PC}_{\mathrm{MAG}}$ are the processing cost of the packet in a simple router, LMA or MAG, respectively.

As mentioned above, the cost of a packet sent to the network is proportional to the distance in hops between source and destination nodes. In Equations 4 and 5, the size of the messages sent to the network is denoted by $S(\mathrm{PBU})$ and $S(\mathrm{PBA})$, respectively, and the distance is represented by $d_{\text {MAG-LMA }}$ and $d_{\text {LMA-MAG }}$ which are the number of hops between the LMA and the MAG, respectively.

The signalling cost of PMIP-MPLS can be formulated as follows:

$$
\begin{aligned}
C_{u}(\mathrm{PMIP}-\mathrm{MPLS})= & N_{h v}[C(\mathrm{PBU})+C(\mathrm{PBA})+C(\mathrm{PATH}) \\
& +C(\mathrm{RESV})]
\end{aligned}
$$

Equation 6 explains the behaviour of the PMIP-MPLS handover mechanism described in [13]. In this approach, when a handover occurs, a PBU message is sent from the MAG to the LMA and a PBA message is expected to be received as the acknowledgement of the binding update in the LMA.

This protocol does not use an IP-in-IP tunnel to forward the packets in the network. An MPLS-based LSP tunnel is created from the LMA to the MAG to forward all the traffic sent to the MN. To signal this tunnel, a PATH message is sent to the network and a RESV message is received to notify the tunnel set-up. $C(\mathrm{PATH})$ and $C(\mathrm{RESV})$ are the cost of the signalling messages and can be expressed as follows:

$$
\begin{aligned}
C(\mathrm{PATH})= & \alpha\left(S(\mathrm{PATH}) d_{\mathrm{MAG}-\mathrm{LMA}}\right) \\
& +\left(d_{\mathrm{MAG}-\mathrm{LMA}}-1\right) \mathrm{PC}_{r}+\mathrm{PC}_{\mathrm{LMA}} \\
C(\mathrm{RESV})= & \alpha\left(S(\mathrm{RESV}) d_{\mathrm{LMA}-\mathrm{MAG}}\right) \\
& +\left(d_{\mathrm{LMA}-\mathrm{MAG}}-1\right) \mathrm{PC}_{r}+\mathrm{PC}_{\mathrm{MAG}}
\end{aligned}
$$

Where $S(\mathrm{PATH})$ and $S$ (RESV) are the size of the PATH and RESV messages, respectively.

Finally, the IPM-TP signalling cost can be derived as:

$$
\begin{aligned}
C_{u}(\mathrm{IPM}-\mathrm{TP})= & \left(N_{h v}-N_{f}\right)\left[2 \mathrm{su}+C\left(\mathrm{PATH}_{\mathrm{Ext}}\right)\right. \\
& \left.+C\left(\mathrm{RESV}_{\mathrm{Ext}}\right)\right]+N_{f}[C(\mathrm{PBU})+C(\mathrm{PBA}) \\
& +C(\mathrm{PATH}) C(\mathrm{RESV})]
\end{aligned}
$$

Equation 9 derives the behaviour of the IPM-TP approach explained in Section 3. In this scheme, when the mobile node detects the handover, it sends a L2 signalling message, whose average size is expressed by su. If the LSP tunnel is extended from previous LMAG to the new LMAG, the cost to signal of this extension is denoted by $C\left(\mathrm{PATH}_{\mathrm{Ext}}\right)$ and $C\left(\mathrm{RESV}_{\text {Ext }}\right)$.

The number of location updates that must be done in a session due to the QoS of a particular flow under an agreed threshold is denoted by $N_{f}$. This value is obtained from $\frac{N_{h v}}{\mathrm{np}}$, where np is the number of path extensions until the QoS requirements are below the threshold.

In the location update, the LMAG notifies the handover to the LLMA by means of the PBU/PBA messages, and a new LSP tunnel is created using RSVP PATH/RESV messages. $C\left(\mathrm{PATH}_{\mathrm{Ext}}\right)$ and $C\left(\mathrm{RESV}_{\mathrm{Ext}}\right)$ expressions can be expressed as follows:

$$
\begin{aligned}
& C\left(\mathrm{PATH}_{\mathrm{Ext}}\right)= \alpha\left(S(\mathrm{PATH}) d_{\mathrm{MAG}-\mathrm{MAG}}\right) \\
&+\left(d_{\mathrm{MAG}-\mathrm{MAG}}-1\right) \mathrm{PC}_{r}+\mathrm{PC}_{\mathrm{MAG}} \\
& C\left(\mathrm{RESV}_{\mathrm{Ext}}\right)= \alpha\left(S(\mathrm{RESV}) d_{\mathrm{MAG}-\mathrm{MAG}}\right) \\
&+\left(d_{\mathrm{MAG}-\mathrm{MAG}}-1\right) \mathrm{PC} r \\
&
\end{aligned}
$$

where $d_{\text {MAG-MAG }}$ is the distance in number of hops between the previous LMAG and new LMAG.

\subsection{Packet delivery cost}

During the active session, the MN communicates with its Correspondent Node $(\mathrm{CN})$ using the bidirectional tunnel established between the MAG and the LMA.

However, in PMIPv6 or PMIP-MPLS protocols, in-flight data packets destined for the MN will be lost in the handover process. In this paper, we only focus on the packet delivery cost during the handover process.

The packet delivery cost is the accumulative traffic overhead caused by packet delivery on the routing paths. That is calculated as the product of the data packet size and the hop distance. 
The packet delivery cost of the PMIPv6 protocol is expressed as:

$$
\begin{aligned}
P_{\text {Cost }}(\mathrm{PMIPv} 6)= & \gamma\left[\beta\left(E(S) S(\text { Data }) d_{\mathrm{MN}-\mathrm{MAG}}\right)+\mathrm{PC}_{\mathrm{MAG}}\right. \\
& +\alpha\left[\left(S(\text { Data })+S\left(\mathrm{TE}_{\mathrm{IP}}\right) d_{\mathrm{MAG}-\mathrm{LMA}}\right]\right. \\
& +\left(d_{\mathrm{MAG}-\mathrm{LMA}}-1\right) \mathrm{PC}_{r}+\mathrm{PC}_{\mathrm{LMA}} \\
& \left.+\alpha\left(S(\text { Data }) d_{\mathrm{LMA}-\mathrm{CN}}\right)+d_{\mathrm{LMA}-\mathrm{CN}} \mathrm{PC}_{r}\right]
\end{aligned}
$$

where $\beta$ denotes the transmission unit cost in the wireless link, $E(S)$ is the average session length in packets and $\gamma$ expresses an increasing rate of expense that is used to indicate the retransmission of the lost packets [25].

The packet delivery of PMIP-MPLS can be derived as follows:

$$
\begin{aligned}
P_{\text {Cost }}(\mathrm{PMIP}-\mathrm{MPLS})= & \gamma\left[\beta\left(E(S) S(\text { Data }) d_{\mathrm{MN}-\mathrm{MAG}}\right)\right. \\
& +\mathrm{PC}_{\mathrm{MAG}}+\alpha[(S(\text { Data }) \\
& \left.\left.+S\left(\mathrm{TE}_{\mathrm{MPLS}}\right) d_{\mathrm{MAG}-\mathrm{LMA}}\right)\right] \\
& +\left(d_{\mathrm{MAG}-\mathrm{LMA}}-1\right) \mathrm{PC}_{r}+ \\
& +\mathrm{PC}_{\mathrm{LMA}}+\alpha\left(S(\text { Data }) d_{\mathrm{LMA}-\mathrm{CN}}\right) \\
& \left.+d_{\mathrm{LMA}-\mathrm{CN}} \mathrm{PC}_{r}\right]
\end{aligned}
$$

Finally, the packet delivery cost of IPM-TP must forward the packets from the LMA to the first MAG which is the head of the tunnel, and this router forwards the packets to the LSP tunnel extended. The packet delivery cost of IPM-TP is formulated as:

$$
\begin{aligned}
P_{\mathrm{Cost}}(\mathrm{IPM}-\mathrm{TP})= & {\left[\beta\left(E(S) S(\text { Data }) d_{\mathrm{MN}-\mathrm{MAG}}\right)\right)+\mathrm{PC}_{\mathrm{MAG}} } \\
& +\left(\alpha\left(S(\text { Data })+S\left(\mathrm{TE}_{\mathrm{MPLS}}\right) d_{\mathrm{MAG}-\mathrm{MAG}}\right)\right. \\
& \left.+\left(d_{\mathrm{MAG}-\mathrm{MAG}}-1\right) \mathrm{PC}_{r}+\mathrm{PC}_{\mathrm{MAG}}\right] \mathrm{np} \\
& +\alpha\left[\left(S(\text { Data })+S\left(\mathrm{TE}_{\mathrm{MPLS}}\right) d_{\mathrm{MAG}-\mathrm{LMA}}\right]\right. \\
& +\left(d_{\mathrm{MAG}-\mathrm{LMA}}-1\right) \mathrm{PC}_{r}+\mathrm{PC}_{\mathrm{LMA}} \\
& +\alpha\left(S(\text { Data }) d_{\mathrm{LMA}-\mathrm{CN}}\right)+\mathrm{PC}_{r} d_{\mathrm{LMA}-\mathrm{CN}}
\end{aligned}
$$

\subsection{Total signalling cost}

Based on the above analysis, the overall signalling cost is obtained as function:

$$
\mathrm{TC}(\cdot)=C_{u}(\cdot)+P_{\text {cost }}(\cdot)
$$

Where the signalling cost $C_{u}(\cdot)$ and $P_{\text {cost }}(\cdot)$ are the signalling cost and packet delivery cost, respectively, for the

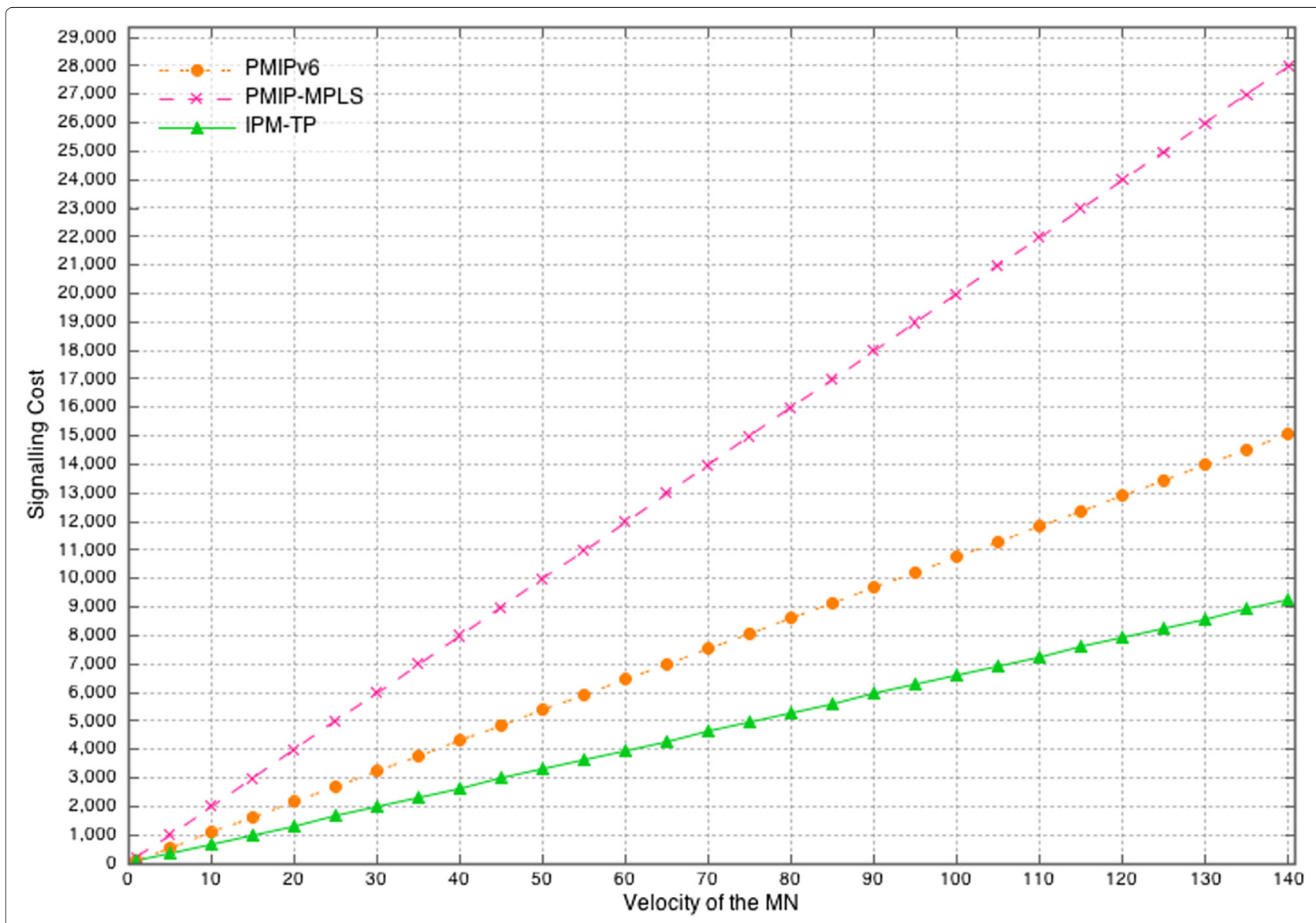

Figure 5 Signalling cost as a function of the velocity of the MN. 
three schemes, i.e. PMIPv6 scheme, PMIP-MPLS scheme and the proposed IPM-TP scheme.

\subsection{Service-blocking probability}

The main role of the LMA is to maintain reachability to the address of the MN while it moves, stores information necessary to associate an MN with its serving MAG, and enables and maintains the relationship between the MAG and LMA. This implies that the traffic sent to the MN is intercepted by the LMA and forwarded using the tunnel to the serving MAG.

Accordingly, the LMA could be in the service-blocking time when all resources of the LMA are busy. This problem has been deeply studied in other fields and works like [26,27].

Therefore, the Erlang B formula has been modified to analyse the service-blocking probability for the LMA. Let $\lambda_{b}$ the packet arrival time to the LMA and $\Delta t$ the packet service time at the LMA. Then, the service-blocking probability $\mathrm{SB}_{\mathrm{LMA}}$ is obtained as follows [28]:

$$
\mathrm{SB}_{\mathrm{LMA}}=\frac{\left.\left(\lambda_{b} / \Delta t\right)^{m}\right) / m !}{\left(\sum_{k=0}^{m}\left(\lambda_{b} / \Delta t\right)^{k}\right) / k !}
$$

where $m$ is the number of resource units for handling packets in the LMA.

\section{Results}

The following parameters are the basic configuration of the topology, mobility, traffic modes and processing cost of the nodes used in the analysis. These parameters are similar to those used in the paper [24]: $R=100 \mathrm{~m}, N=$ $30, \mathrm{np}=5, \mathrm{PC}_{r}=8, \mathrm{PC}_{\mathrm{MAG}}=\mathrm{PC}_{\mathrm{LMA}}=\alpha=1, \beta=1.5$, $v=[1-140] \mathrm{m} / \mathrm{s}, d_{\mathrm{MAG}-\mathrm{LMA}}=6$ hops, $d_{\mathrm{MAG}-\mathrm{LMA}}=$ 20 hops, $d_{\mathrm{MN}-\mathrm{MAG}}=1$ hop and $d_{\mathrm{LMA}-\mathrm{CN}}=25$ hops.

The sizes of the messages are defined as follows: $S(\mathrm{PBU})=76$ bytes, $\mathrm{su}=50$ bytes, $S(\mathrm{PBA})=76$ bytes, $S\left(\mathrm{TE}_{\text {mpls }}\right)=4$ bytes, $S\left(\mathrm{TE}_{\mathrm{IP}}\right)=40$ bytes, $E(S)=14$, $S($ PATH $)=64$ bytes, $S($ RESV $)=64$ bytes, $S($ Data $)=$ 120 bytes, $\lambda_{s}=[0.1-0.7]$.

Figure 5 shows the signalling cost of the underlying protocols. As can be observed, PMIPv6 and PMIP-MPLS have a high-registration cost because of the frequent registration with the LMA to update the binding cache. Any movement done by the $\mathrm{MN}$ must be registered with the LMA, and this increases the overhead in the network. PMIP-MPLS has the higher registration cost because the tunnel LSP is signalized and this increases the signalling

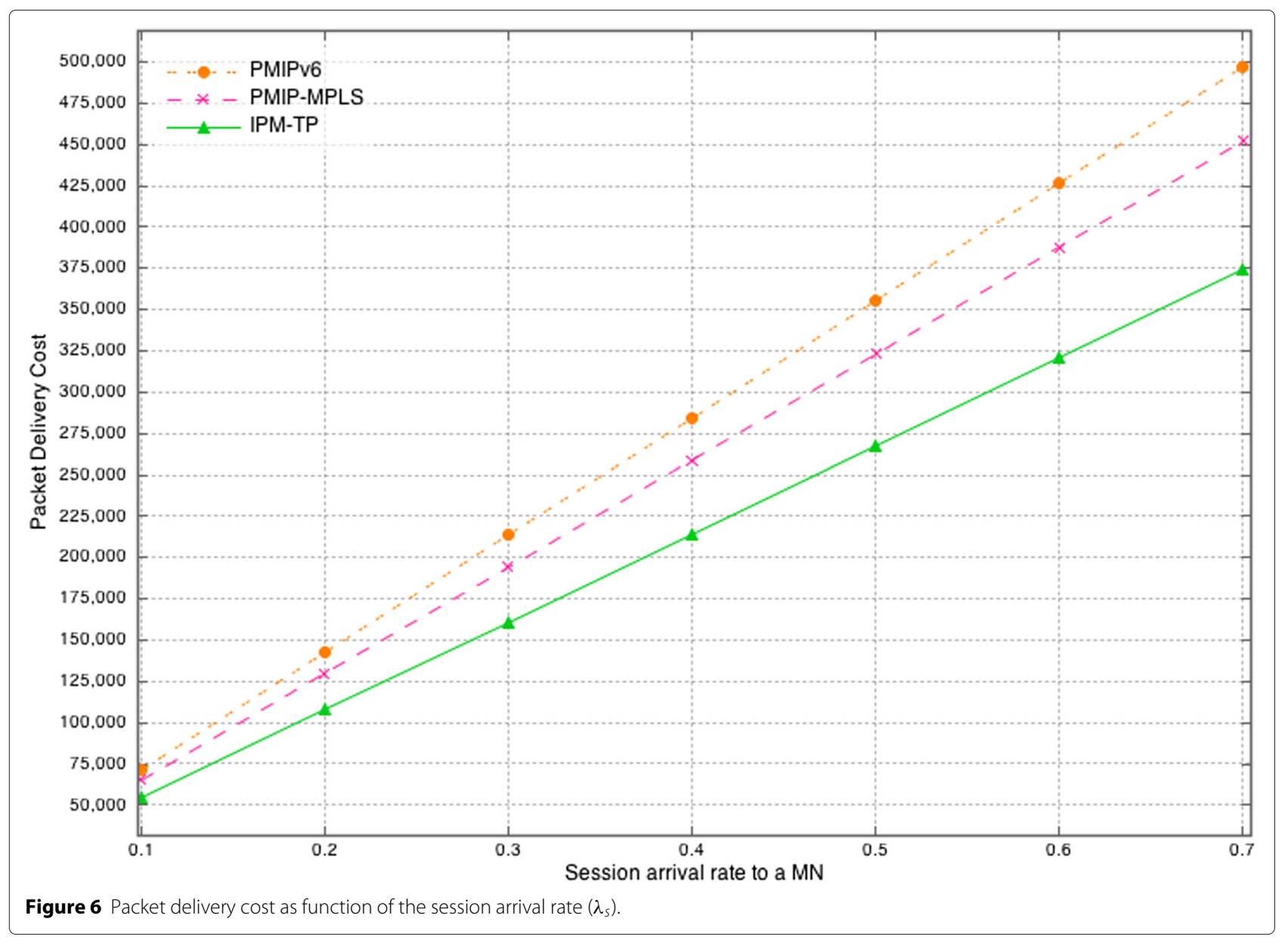


cost. With the IPM-TP approach, the registration overhead is reduced by around 40\% compared with PMIPv6 and $67 \%$ compared with PMIP-MPLS.

In a real scenario, if a mobility management protocol has a high signalling cost, the control plane implemented in the mobility management protocol introduces a high amount of signalling messages. This cost is produced due to the signalling messages sent to the mobility anchor nodes during the handover or due to a frequent signalling to track the mobility of the MN.

As shown in Figure 5, PMIP-MPLS sends a high number of signalling messages (MPLS and mobility-related signalling) to notify the handover of the MN. This higher number of messages affects the handover latency [29], which is the time elapsed from the moment the handoff event is detected to the moment the first packet is received from the new subnet. IPM-TP has a fewer frequency of registration with the LMAG, and therefore, the signalling cost is reduced as well as the handover latency.

Figure 6 shows the packet delivery cost against the session arrival rate. Assuming the session arrival rate varies from 0.1 to 0.7 .
The PMIPv6 and PMIP-MPLS packet delivery cost is higher than IPM-TP because of the packet loss during a handoff. This value is increased in both protocols due to the packet retransmission.

The IPM-TP approach avoids the packet loss because the L2 trigger signals the movement and can reroute the packet in the packet core network to the new LMAG which is going to serve the MN.

This LMAG will also implement a buffering technique to store in-flight packets. When the $\mathrm{MN}$ is attached to the new LMAG, all the packets will be delivered to the MN. As can be observed in Figure 6, packet delivery cost is lower with this approach.

In a real scenario, if a mobility management protocol has a high packet delivery cost, the forwarding plane introduces overhead to the network when the packets are sent or received using the tunnelling mechanism.

As shown in Figure 6, PMIPv6 introduces higher overhead to the communication due to the tunnelling mechanism and the packet loss. The IP in the IP tunnelling mechanism increases the overhead introduced in the network ( 20 bytes of tunnelling header), and this implies in

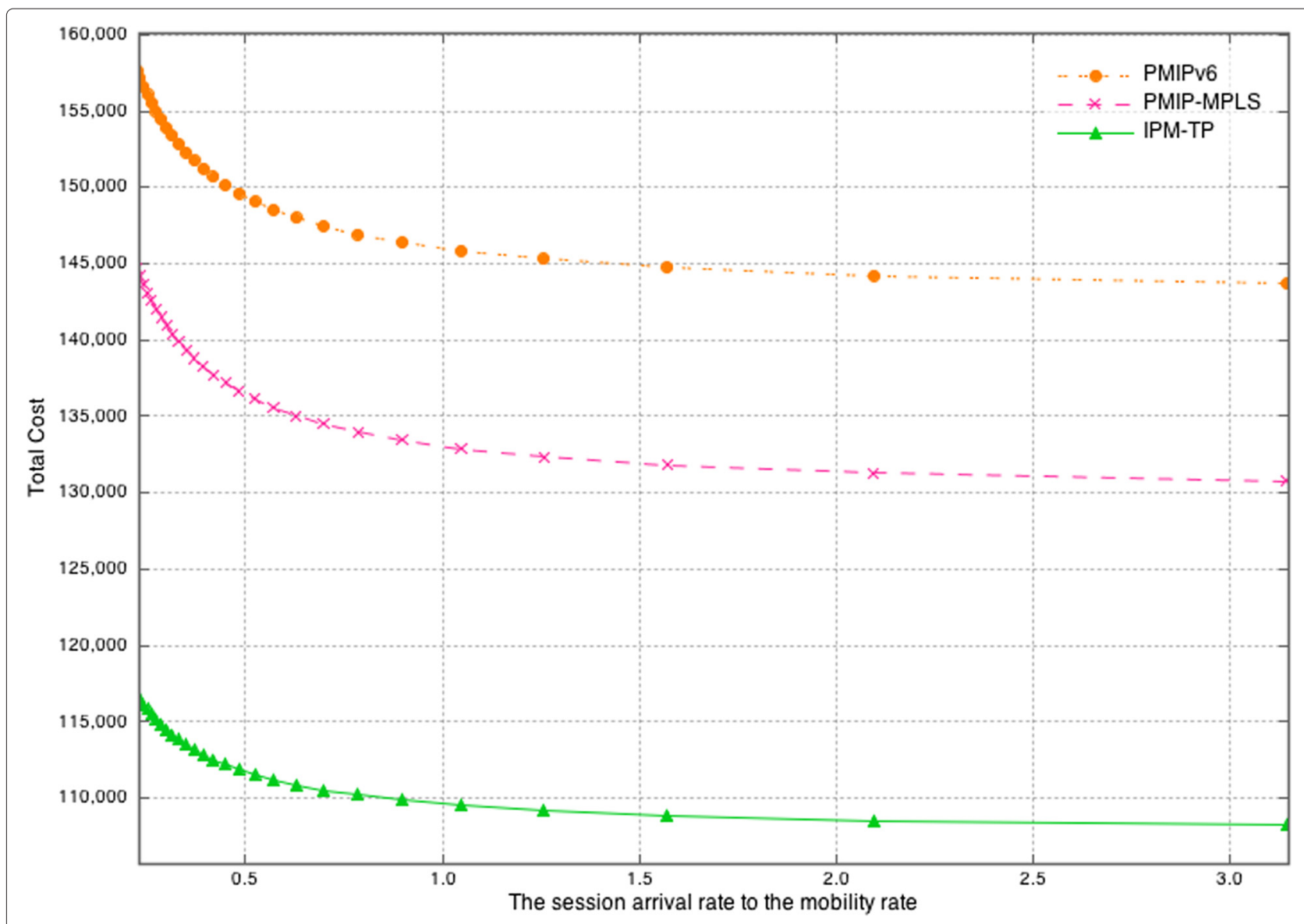

Figure 7 Total cost in function of the session arrival rate (SMR). 
a real scenario a higher probability of network congestion which could increase the packet loss in the backhaul or the BS. IPM-TP decreases the overhead using MPLS-TP tunnelling mechanism ( 4 bytes of tunnelling header), and also, the buffering mechanism reduces the packet loss in the network produced during the $\mathrm{MN}$ handovers.

The total cost is presented in Figure 7. It is presented as a function of SMR. The SMR is the ratio of the session arrival rate to the mobility rate (i.e. subnet-crossing rate). In the mobility model, the SMR is defined as SMR = $\frac{\lambda_{s}}{\mu_{c}}$ [19]. Note that a higher value of SMR indicates low mobility; thus, the low signalling update cost occurs. The value of $\lambda_{s}$ is 0.2 , and the $v$ is varied from 10 to $120 \mathrm{~m} / \mathrm{s}$.

As can be observed in Figure 7, as SMR increases, the $\mathrm{TC}(\cdot)$ decreases for all the protocols. This is because the mobility of the mobile node decreases and the signalling cost and packet delivery cost decrease too. The total cost of PMIPv6 is the higher cost because of the IP-in-IP tunnelling mechanism of PMIPv6.

PMIP-MPLS decreases the total cost of PMIPv6 because of the tunnelling mechanism. With the MPLS tunnel, the overhead added by the tunnelling method decreases due to the MPLS label size, which is 15 times less than traditional IP-in-IP tunnel.

Finally, with IPM-TP architecture, the original LSP tunnel is extended to forward all in-flight packets and buffer them in the new LMAG. With this mechanism, the total cost is lower than PMIP-MPLS because, with this protocol, all in-flight packets are lost when a handover is produced.

Figure 8 depicts the service-blocking probability of the LMA. Assuming that $m$ and $\Delta t$ are 10 and 1 , respectively. Then, the traffic intensity of LMA, $\rho=\frac{\lambda_{b}}{\Delta t}$, is expressed as follows:

$$
\rho= \begin{cases}N_{h v}+\left(E(S) \lambda_{s}\right) & \text { PMIP / PMIP-MPLS } \\ N_{f}+\left(E(S) \lambda_{s}\right) & \text { IPM-TP }\end{cases}
$$

As could be observed in Figure 8, the IPM-TP architecture provides a lower service-blocking probability because of the tunnel extension mechanism.

This mechanism reduces the signalling and the creation of new tunnels to the LMA when a handoff is produced. PMIPv6 and PMIP-MPLS create new tunnels between the new MAG and the LMA, and the new tunnel creation increases the probability to block the service in the LMA.

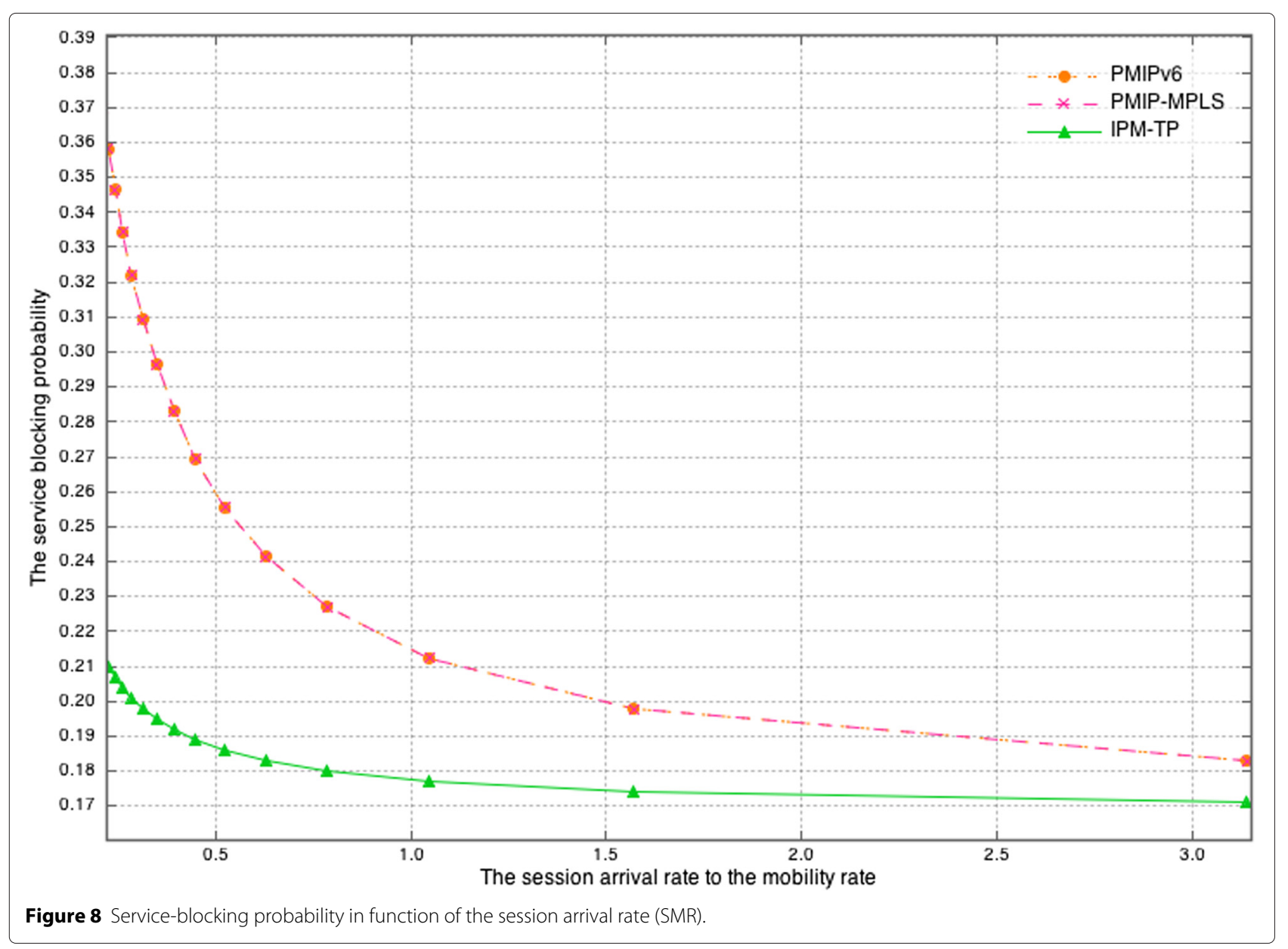


In addition, comparing IPM-TP with traditional PMIPv6-based protocols, IPM-TP provides the best performance in cost consumption and service-blocking probability due to the tunnel extension mechanism which reduces the signalling and the packet loss when a handover is produced.

\section{Conclusions}

This article presents a novel architecture called Integrated Proxy Mobile MPLS-TP, which supports seamless mobility management in HetNets, traffic engineering and QoS and encourages network convergence.

The architecture presented is a simple and packet-based backhaul solution which merges traditional fixed and mobile networks and enables the integration of heterogeneous networks and fixed networks which interconnects these small cells, and this is one of the providers' main open issues.

With IPM-TP, the QoS offered by the network to the mobile nodes improves the reduction in signalling costs, packet delivery cost when a handoff is produced and also the probability to block a service by the LMA. Therefore, packet loss is avoided. To demonstrate this, a comparison between the proposed IPM-TP architecture and existing solutions (PMIPv6 and PMIP-MPLS solutions) was made.

In this work, we have presented a mobility management protocol which provides seamless mobility with traffic engineering and QoS to HetNets. Extending this work, we will investigate the behaviour of the mobility management protocol under real user mobility traces which allows studying the robustness of the protocol with a higher number of MN and different types of microcells.

\section{Competing interests}

The authors declare that they have no competing interests.

\section{Acknowledgements}

This work has been financed in part by the European Regional Development Fund through the project SOE4/P3/E804, 'Federation Infrastructure for Virtual Desktop Infrastructure'.

\section{Author details \\ ${ }^{1}$ Research, Technological Innovation and Supercomputing Center of Extremadura (CénitS), National Road 521, km 41.8, Postal code 10.071 Cáceres, Spain. ${ }^{2}$ Department of Computing and Telematics System Engineering, GITACA Research Group, University of Extremadura, University Avenue, Postal code 10.003 Cáceres, Spain.}

Received: 8 July 2014 Accepted: 24 March 2015

Published online: 23 April 2015

\section{References}

1. Cisco Systems Inc., Cisco visual networking index: global mobile data traffic forecast update 2012-2017 (2013). [Online]. Available:www.cisco. com. Access date: April 2015

2. BA Bjerke, LTE-advanced and the evolution of LTE deployments. Wireless Commun. IEEE. 18(5)، 4 (2011). doi:10.1109/MWC.2011.6056684
3. IF Akyildiz, DM Gutierrez-Estevez, EC Reyes, 2010. The evolution to 4G cellular systems: LTE-Advanced. Phys. Commun. 3, 217-244 (2010)

4. S Frattasi, H Fathi, F Fitzek, R Prasad, M Katz, Defining 4G technology from the users perspective. IEEE Network. 20(1), 35-41 (2006). doi: 10.1109/MNET.2006.1580917

5. P Makris, N Nomikos, D Skoutas, D Vouyioukas, C Skianis, J Zhang, C Verikoukis, A context-aware framework for the efficient integration of femtocells in IP and cellular infrastructures. EURASIP J. Wireless Commun. Netw. 2013, 62 (2013)

6. S Gundavelli, K Leung, V Devarapalli, K Chowdhury, B Patil, Proxy Mobile IPv6, RFC 5213 (2008)

7. ETSI, Technical specifications of Universal Mobile Telecommunications System (UMTS); LTE; Proxy Mobile IPv6 (PMIPv6) based mobility and tunnelling protocols; Stage 3 (3GPP TS 29.275 version 11.4.0 Release 11) (2012)

8. The Broadband Forum, Technical specifications for interworking between next generation fixed and 3GPP wireless networks TR-203 (2012)

9. S Namal, M Liyanage, A Gurtov, Realization of mobile femtocells: operational and protocol requirements. Wireless Personal Commun 71(1), 339-364 (2013)

10. Z Ghebretensa, J Harmatos, K Gustafsson, Mobile broadband backhaul network migration from TDM to carrier Ethernet. IEEE Commun. Mag. 48(10), 102-109 (2010)

11. O Tipmongkolsilp, S Zaghloul, A JukanFrattasi, The evolution of cellular backhaul technologies: current issues and future trends. IEEE Commun. Surv. Tutorials. 13(1), 97-113 (2011)

12. The Broadband Forum, Technical specifications for MPLS in mobile backhaul networks, Tech. Rep. TR-221, The Broadband Forum (2011)

13. F Xia, Sarikaya B, MPLS tunnel support for Proxy Mobile IPv6, Internet-Draft (2008)

14. R Winter, The coming of age of MPLS. IEEE Commun. Mag. 49(4), 78-81 (2011)

15. E Bellagamba, L Andersson, P Skoldstrom, D Ward, Takacs A, Configuration of pro-active Operations, Administration, and Maintenance (OAM) functions for MPLS-based transport networks using RSVP-TE, Draft IETF (2012)

16. IF Akyildiz, W Wang, A dynamic location management scheme for next-generation multitier PCS systems. Wireless Commun. IEEE Trans. 1(1), 178-189 (2002)

17. J Ho, SM Akyildiz, F lan, Mobile user location update and paging under delay constraints. Wireless Networks. 1(4), 413-425 (1995)

18. G Morales-Andres, M Villen-Altamirano, in Proceedings of the Forum Telecom 1987. An approach to modelling subscriber mobility in cellular radio networks (Geneva, Switzerland, 1987), pp. 185-189

19. S Pack, T Kwon, Y Choi, A performance comparison of mobility anchor point selection schemes in hierarchical mobile IPv6 networks. Comput Networks. 51(6), 1630-1642 (2007)

20. KXu, Tipper D, P Krishnamurthy, Q Yi, An efficient hybrid model and dynamic performance analysis for multihop wireless networks. Comput Networking Commun. (ICNC), 2013 Int Conference 1090-1096 (2013)

21. B Liang, ZJ Haas, Predictive distance-based mobility management for multidimensional PCS networks. IEEE/ACM Trans. Netw. 11, 718-732 (2003)

22. C-G Vicente, V Pla, P Escalle-García, in Network Performance Engineering Mobility Models for Mobility Management (Springer, Berlin Heidelberg, 2011), pp. 716-745

23. FV Baumann, IG Niemegeers, in Proceedings of IEEE International Conference on Universal Personal Communications (UPC) 1994. An evaluation of location management procedures (San Diego, USA, 1994), pp. 359-364

24. C Makaya, S Pierre, An analytical framework for performance evaluation of IPv6-based mobility management protocols. IEEE Trans. Wireless Commun. 7(3), 972-983 (2008)

25. K Lee, Y Mun, in Proc. ICCSA. An efficient macro mobility scheme supporting fast handover in hierarchical mobile IPv6, vol. 3480, (2005), pp. 408-417

26. K Ayyappan, K Narasimman, P Dananjayan, in Proceedings of Future Computer and Communication, 2009. ICFCC 2009. Int. Conference. SINR based vertical handoff scheme for QOS in heterogeneous wireless networks, vol. 1, (2009), pp. 117-121

27. A Jabban, Y Nasser, M Helard, in Proc. Telecommunications (ICT), 2012 19th Int Conference. SINR based network selection strategy in integrated 
heterogeneous networks, vol. 1, (2012), pp. 1-6.

doi: 10.1109/ICTEL.2012.6221276

28. J-W Lin, J Arul, An efficient fault-tolerant approach for mobile IP in wireless systems. IEEE Trans. Mob. Comput. 2(3), 207-220 (2003)

29. J-H Lee, J-M Bonnin, I You, T-M Chung, Comparative handover performance analysis of IPv6 mobility management protocols. Ind. Electron. IEEE Trans. 60(3), 1077-1088 (2013)

Submit your manuscript to a SpringerOpen ${ }^{\circ}$ journal and benefit from:

- Convenient online submission

- Rigorous peer review

- Immediate publication on acceptance

- Open access: articles freely available online

- High visibility within the field

- Retaining the copyright to your article

Submit your next manuscript at $\boldsymbol{\triangleright}$ springeropen.com 